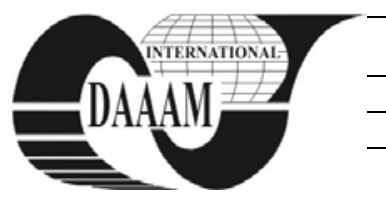

\title{
ROBOT PROGRAMMING FOR SURFACE SCANNING BASED ON DATA FROM PHOTOGRAMMETRY
}

\author{
KONIECZNY, R[oman]
}

\begin{abstract}
The paper presents a general concept of the automated three-dimensional scanning technology that uses structured light projection. During the measurement, the scanner is moved and positioned at specified points using a robot. The initial geometric model representing the scanned object, generated as a result of the photogrammetric measurement process, is used as input data for robot control program creation. This rough model is analyzed by developed application, which calculates positions for the industrial robot with a fitted scanner. The described procedure were tested during measurements of car body parts
\end{abstract}

Key words: reverse engineering, photogrammetry, 3D scanning, measurements automation, robot programming

\section{INTRODUCTION}

Digital representations required in the different stages of the product development process are often obtained using reverse engineering (RE) methods. This technique can also be applied for digitalization of a physical conceptual model created by a visual designer. Reverse engineering in this case is an integral part of the product development process (Sokovic \& Kopac, 2006).

Techniques most frequently used for digitalization include optical scanning with the use of structured light or laser scanning (Cheng et al., 2010), (Kus, 2009). Both methods are very precise, with up to $0.02 \mathrm{~mm}$ accuracy, but also timeconsuming (despite a short time of single measurement lasting a few seconds only). When scanning large objects, such as automobile body is necessary to perform multiple measurements from multiple angles, which in turn lengthens the whole process up to several hours.

In the proposed methodology, the coordinates of the next scanner settings necessary for the proper collection of information about geometric shapes of test object are determined based on an approximate geometric model. This model is obtained using the fast photogrammetric measurements. Based on such a set of coordinates a control program for industrial robot, which moves the scanner performing a series of measurements, is created.

There are ready-to-use systems in which the scanner is coupled with the robot. However, these systems are only used as coordination systems to determine the spatial position of the scanner based on the available CAD model, and to compare the model generated by the scanning process with the CAD model (GOM, 2010).

\section{THREE-DIMENSIONAL SCANNING}

The scanner's projector projects a pattern of stripes of known density on a given object. Straight lines are getting distorted adequately to the deformation of the object surface, and the image is recorded on matrixes of two cameras. Using the input data (the structure of light, the camera recorded image, the calibration parameters, and the angle between direction of projection and direction of reading) coordinates are calculated for each pixel of the camera. The result of single measurement is a cloud of points whose number is directly dependent on the resolution of cameras (Cheng et al., 2010).

Engineering photogrammetry is an alternative method for obtaining three-dimensional data. It is used especially for measuring large objects or for fast inspection of object location (Clarke \& Wang, 2000), (Luhmann et al., 2007).

Photogrammetric measurements allow generating a cloud of reference points representing a given object, on the basis of appropriately taken series of photographic pictures. Specific markers are used in close range photogrammetry, some of them are positioned on the measured object (markers), others are positioned in its vicinity (code stamps), allowing to orient the created pictures against each other. Additionally, scaling rods must be positioned on the photographed scene, to re-scale the produced cloud of points to their actual size.

\section{AUTOMATED SCANNING METHODOLOGY}

The prepared object is photographed from different camera positions. The received images are analyzed and, thanks to superposition of data from many pictures, three-dimensional data are generated in the form of a cloud of reference points, representing markers placed on the object. The coordinates of points are sent to the Catia V5 software, where a mesh of triangles is spread and later saved in STL format. This representation gives the coordinates of the vertexes of a mesh of triangles and the coordinates of the normal vectors of each triangle, determining the outward and inward facing surfaces. The data in text format are imported by the designed application. In the first phase of the algorithm, the whole object is divided into smaller sectors of sizes directly depending on the size of the scanner measurement field.

The measurement field is a surface. However, considering the scanner's depth of focus, it has been assumed that triangles included in a cube, defined according to the measurement field, will be searched.

After defining the length of the cube side and its position in space, a filter is created, which searches data in the STL file for all triangles occurring in the area of the cube. A set of selected triangles also includes those, which are only fragmentarily present in the set space. In the next step, the surface area of those triangles is calculated, and the weighting factor, which is proportional to the area, is determined.

Considering this factor, and based on the normal vectors of the respective triangles, an averaging direction vector is calculated for the analyzed fragments of the surface. Its origin is determined by averaging the coordinates of the centres of the triangles located in the analyzed set. An additional condition, verified in the described procedure, analyzes the values of angle deviations of every normal vector of the respective triangles, from the direction of the resultant vector.

This follows from the fact that the scanner has a maximum "angle of observation" to the examined surface, allowing it to scan properly. If, for a given triangle, the deviation of angles is 
higher than the adopted acceptable value and the percentage share of its surface area in the surface area of the considered fragment of solid exceeds the declared threshold value, its data, together with data of other triangle failing to meet the conditions, are saved in a separate file. The data are again loaded by the application and, determine the positions of additional scanning procedures, as soon as the analysis of other areas is finished.

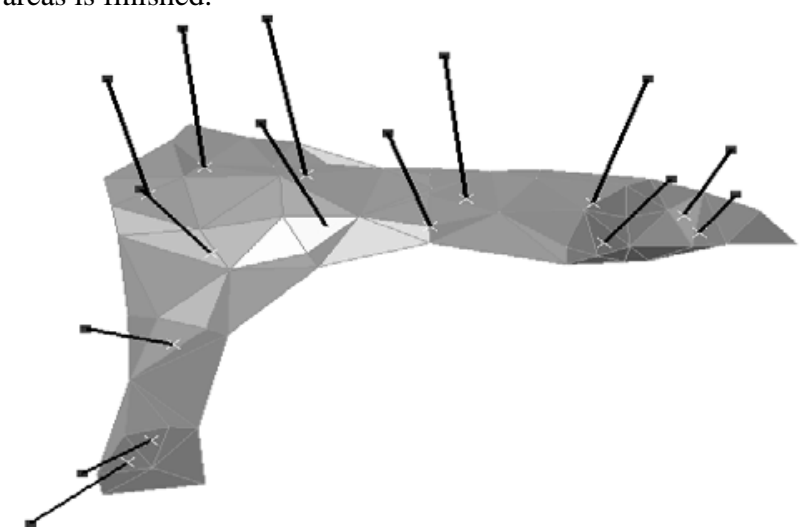

Fig. 1. Rough fender model and scanner spatial positions generated by designed application

For every required measurement, the position of the scanner is determined on the axis of the resultant vector, within a previously defined distance, which ensures a correct scanning process. Figure 1 shows the positions of scanner, as they have been calculated by the presented algorithm for the scanned fender.

\section{SCANNING TESTS}

The described tests have been carried out using the Kuka KR30 industrial robot equipped with a specially designed and produced GOM Atos optic scanner holder, GOM Tritop photogrammetric measurement system and the software developed by the authors.

The Kuka KR30 industrial robot is responsible for moving the scanner to the next set position. In order to minimize the total scanner travel and the scanning time, there is a procedure used in the application, scheduling the points of scanning with the use of a genetic algorithm.

To be able to position the scanner precisely, the robot must have its global coordinate system linked with a local system determined by the calibration cross. In addition, the tool - the scanner - must be calibrated.

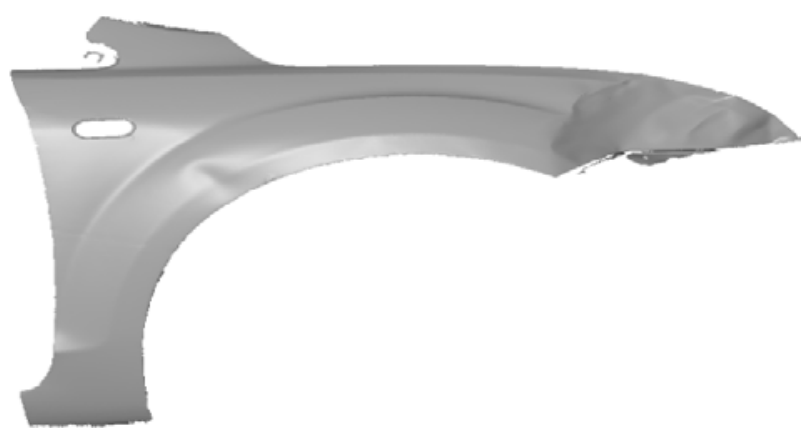

Fig. 2. 3D fender model scanned using the described method

Before the final creation of the robot control program, its planned movements are visualized by loading paths geometry data into the CATIA system. This method allows protecting against possible scanner collisions. Points generated by the application are converted into robot coordinates, using a homogenous transformation matrix typical for the robot controller.

After the set scanner position is reached, the single scanner shot is started remotely. The scanning procedure produces a 3D fender model in the form of a triangle mesh. Figure 2 presents the model generated by automated scanning. Some missing surface fragments can be seen in hard-to-reach places. They result from the robot's constrained operation area, limiting the size of the scanned object and access to points, which require the actuator to be rotated out of the range of the axis of rotation. Large-size objects can be moved against the robot, maintaining the global coordinate system in the same position, with calculations made again in the application.

\section{CONCLUSIONS AND FUTURE WORK}

An innovative concept of the automation of a threedimensional scanning process using structured light projection technology has been presented, along with its implementation using well-established industrial equipment and control software. The significant shortening of the entire scanning procedure, which results from cutting time needed by the operator to move the scanner to the next position, is undoubtedly the biggest advantage of the described method.

In the presented example of the fender, it took the operator almost an hour to scan the fender using a scanner fitted on a stand, while it took only about 10 minutes to scan it using the robot. The labour intensity required for preparing the scanner and the object for the scanning procedure is similar in both cases (positioning markets, tarnishing, selecting the appropriate field of measurement, calibrating the scanner).

The described method requires additional photogrammetric measurements, but these take a little time only, thanks to the online analysis of the consecutive pictures straight after sending them to the system (less than 10 minutes in case of the fender).

The authors are working further on the described method to use a turntable allowing to apply the entire scanning procedure for medium sized objects and thus to extend its functionality.

\section{ACKNOWLEDGMENTS}

This work has been financially supported by the Polish Ministry of Science and Higher Education, research project no. 1478/B/T02/2009/36.

\section{REFERENCES}

Cheng F.-H., Lu Ch-T., Huang Y.-S. (2010). 3D Object Scanning System By Coded Structured Light, Third International Symposium on Electronic Commerce and Security, ISBN 9781424482313, Guangzhou, China, p. 213-217

Clarke T., and Wang X. (2000). The Control of a Robot EndEffector using Photogrammetry", International Archives of Photogrammetry and Remote Sensing, Vol 33 (B5), ISSN: 1682-1750, Amsterdam, p. 137-142

Kus A. (2009). Implementation of $3 D$ Optical Scanning Technology for Automotive Applications, Sensors, 9, ISSN 1424-8220, p. 1967-1979

Luhmann T., Robson S, Kyle S., Harley I. (2007). Close Range Photogrammetry: Principles, Techniques and Applications, ISBN 0-4701-0633-6, Wiley,

Sokovic, M.; Kopac, J. (2006). Reverse Engineering as Necessary Phase by Rapid Product Development, Journal of Materials Processing Technology Vol. 175, ISSN: 09240136, p. 398-403

*** (2011). Automated Robot Inspection Cell for Quality Control on Sheet Metal Components, http://www.gom.com, Accessed on: 2011-06-10 